\title{
APPROXIMATION OF THE ORIENTATION DISTRIBUTION OF GRAINS IN POLYCRYSTALLINE SAMPLES BY MEANS OF GAUSSIANS
}

\author{
D. I. NIKOLAYEV and T. I. SAVYOLOVA \\ MIFI (Moscow Engineering Physical Institute), Kashirskoye shosse 31, Moscow \\ 115409, USSR \\ K. FELDMANN \\ Zentralinstitut fur Kernforschung Rossendorf, PSF 19, Dresden 8051, GDR
}

(Received April 19, 1990; in final form November 30, 1990)

\begin{abstract}
The orientation distribution function (ODF) obtained by classical spherical harmonics analysis may be falsified by ghost influences as well as series truncation effects. The ghosts are a consequence of the inversion symmetry of experimental pole figures which leads to the loss of information on the "odd" part of ODF.

In the present paper a new method for ODF reproduction is proposed. It is based on the superposition of Gaussian distributions satisfying the central limit theorem in the $\mathrm{SO}(3)$-space as well as the ODF positivity condition. The kind of ODF determination offered here is restricted to the fit of Gaussian parameters and weights with respect to the experimental pole figures. The operating mode of the new method is demonstrated for a rolling texture of copper. The results are compared with the corresponding ones obtained by the series expansion method.
\end{abstract}

KEY WORDS ODF calculation, Ghost correction, Gaussian functions.

\section{INTRODUCTION}

The most widely applied method to solve the reproduction problem of the ODF from experimental pole figures is the series expansion formalism proposed by Bunge (1965). In its originally used version it was not able to avoid regions of negative values and ghost effects in the ODF, caused by series termination and the missing of information on the odd part of the ODF (Matthies, 1979). Now, the zero range method (Bunge and Esling, 1979) and more recently the positivity method (Dahms and Bunge, 1989) permit to take into account the odd part of the ODF also.

Another method to eliminate the mentioned phenomena has been proposed by Luecke et al., 1981. They determine the even part of the ODF using the series expansion technique. The true ODF is assumed to be constructed by the minimum number of Gaussians fitted to the spherical harmonics function and takes into account the characteristics of the experimental pole figures. 
In the present paper a new method for ODF determination from experimental data is proposed. The ODF is represented by Gaussians the parameters of which are fitted in the pole figure space directly using the known analytical relationships between ODFs and pole figures formed by such type of distributions.

The way of calculations is illustrated for cubic lattice symmetry. It can be generalized to other symmetries as well.

The application of the proposed method is demonstrated for the example of the rolling texture of copper. The results are compared with the corresponding data obtained from spherical harmonics analysis.

\section{ODF REPRESENTATION BY $\delta$-FUNCTIONS (DISCRETE CASE)}

The solution of the reproduction problem from pole figures of one single crystal is discussed by Matthies (1981) taking into account various symmetries. In this paper the corresponding mathematical formalism has been developed for a polycrystalline sample consisting of a finite number of grains with cubic, hexagonal or orthorhombic lattice symmetry, respectively (T. I. Bukharova and T. I. Savyolova, 1985; T. I. Savyolova, 1985; D. I. Nikolaev and T. I. Savyolova 1987).

If there is a limited number $N$ of grains in the specimen the corresponding ODF can be represented as a linear combination of $\delta$-functions:

$$
f(g)=\sum_{i=1}^{N} a_{i} f_{i}\left(g, g_{i}\right)
$$

where $a_{i}>0$ is the size-induced weight factor of the given grain and $f_{i}$ is a $\delta$-function with the mass at point $g_{i} \in \mathrm{SO}(3)$. In the case of cubic lattice symmetry the function $f(g)$ in Eq. (1) can be shown to be determinable from one pole figure in an unique way.

Let $G_{B}=\left\{g_{B i}\right\}, i=1,2, \ldots, N$ be the subgroup of the single crystal symmetry in the $\mathrm{SO}(3)$-space. For cubic lattices $G_{B}$ consists of 24 elements.

The set of all unit vectors being normal to one of the lattice planes of a selected family $\{h k l\}$ is named as the vector star $\{h k l\}$. The number of unit vectors of a given vector star is equal to the multiplicity. In this way, the $\{001\}$ vector star consists of 6 vectors (the $\{111\}$ one of 8 and the $\{110\}$ vector star of 12 vectors, respectively). Three of the $\{001\}$ vectors are orthogonal to each other, the others are oppositely directed to the first ones. This vector star corresponds to the $\{001\}$ pole figure of the single crystal.

Problem A: Let the vector star $I$ be given. Then, all transformations $g \in \mathrm{SO}(3)$ have to be found in a way, that

$$
\left\{g \cdot h_{i}\right\}=I, \quad i=1,2, \ldots, k .
$$

Solution: The transformation $g^{*}$ is determined unambiguously, if the vectors $\mathbf{y}_{1}, \mathbf{y}_{2}$ are chosen from the vector star $I$ as an ordered pair performing the condition $\left(\mathbf{y}_{1}, \mathbf{y}_{2}\right)=0$ in the case of $\{001\}$ with $g^{*} \cdot \mathbf{h}_{i}=\mathbf{y}_{1}$ and $g^{*} \cdot \mathbf{h}_{2}=\mathbf{y}_{2}$. All other rotations being solutions of problem $A$ are obtained from $g^{*}$ by multiplication from the left side with $g_{B i} \in G_{B}$. 
The effect of any rotating operation $g^{*} \in S O(3)$ on the vectors $h_{1}, h_{2},\left[h_{1}, h_{2}\right]$ is well-known. The mapping of the vectors as a result of the rotating operation $g^{*}$ is:

$$
\begin{aligned}
& \mathbf{h}_{1}=(100) \stackrel{g^{*}}{\longrightarrow} \mathbf{y}_{1}=\left(y_{1}^{1}, y_{1}^{2}, y_{1}^{3}\right) \\
& \mathbf{h}_{2}=(010) \stackrel{g^{*}}{\longrightarrow} \mathbf{y}_{2}=\left(y_{2}^{1}, y_{2}^{2}, y_{2}^{3}\right) \\
& \mathbf{h}_{3}=\left[\mathbf{h}_{1}, \mathbf{h}_{2}\right]=(001) \stackrel{g^{*}}{\longrightarrow}\left[\mathbf{y}_{1}, \mathbf{y}_{2}\right]=\left(\left[\mathbf{y}_{1}, \mathbf{y}_{2}\right]^{1},\left[\mathbf{y}_{1}, \mathbf{y}_{2}\right]^{2},\left[\mathbf{y}_{1}, \mathbf{y}_{2}\right]^{3}\right)
\end{aligned}
$$

These vectors correspond to the traces from equivalent lattice plane normals of a cubic single crystal in the pole figure. Now the desired rotation $g^{*}$ can be written as an orthogonal matrix

$$
g^{*}=\left(\begin{array}{lll}
y_{1}^{1} & y_{2}^{1} & {\left[\mathbf{y}_{1}, \mathbf{y}_{2}\right]^{1}} \\
y_{1}^{2} & y_{2}^{2} & {\left[\mathbf{y}_{1}, \mathbf{y}_{2}\right]^{2}} \\
y_{1}^{3} & y_{2}^{3} & {\left[\mathbf{y}_{1}, \mathbf{y}_{2}\right]^{3}}
\end{array}\right)
$$

The determination of $g^{*}$ for other pole figures can be performed in an analogous manner (see appendix).

Problem B: There is a finite set of unit vectors $Z$ corresponding to the chosen kind of vector stars of all grains under discussion and a function $r$ with the property

$$
Z \stackrel{r}{\longrightarrow} R^{+}
$$

where $R^{+}$represents the set of non-negative numbers. The function $r$ corresponds to one of the pole figures related to an ODF as described by Eq. (1). Now a finite set of rotations $\left\{g_{1}, g_{2}, \ldots, g_{N}\right\} \in \mathrm{SO}(3)$ and a function $R$ have to be found with

$$
\left\{g_{1}, g_{2}, \ldots, g_{N}\right\} \stackrel{R}{\longrightarrow} R^{+}
$$

possessing the following properties:

$-U\left\{g_{j} \cdot h_{i}\right\}=\left\{\left.g_{j} \cdot h_{i}\right|_{j=1,2, \ldots, N ; i=1,2, \ldots, k}\right\}=Z$, i.e. the set $Z$ represents the conjunction of vector stars corresponding to the $N$ grains of the polycrystalline sample.

-the following relationship is valid for any $z \in Z$

$$
\sum_{\left\{g_{j} \cdot z \in\left\{g_{j} \cdot \mathbf{h}_{i}, i=1,2, \ldots, k\right\}\right\}} R\left(g_{j}\right)=r(z)
$$

Eq. (7) permits to determine the weights $a_{i}$ in an ODF as described in Eq. (1) by means of the function $r(z)$. This is equivalent to the representation of rotation $g_{j}$ to within the transformation $g_{B i} \in G_{B i}$ corresponding to the given pole figures for $\mathbf{h}_{i}, i=1,2, \ldots, k$.

Solution of problem B: The set $Z$ has to be split up into vector stars $I_{j}$. From each vector star $I_{j}$ an ordered pair of vectors $\mathbf{y}_{1 j}$ and $\mathbf{y}_{2 j}$ is selected which corresponds to the vectors $h_{1}$ and $h_{2}$. The desired rotation $g_{j}$ is unambiguously 
determined by the conditions $\mathbf{y}_{1 j}=g_{j} \cdot \mathbf{h}_{1}$ and $\mathbf{y}_{2 j}=g_{j} \cdot \mathbf{h}_{2}$. The function $R$ is uniquely found from the equation system (7). Every matrix $g_{j}$ has the form of formula (4) and is determined in an unambiguous way to within the multiplication from the left to one of the matrices from $G_{B}$.

Model investigations have been performed concerning the direct and the reverse problem in order to verify the described formalism. From an ODF of type Eq. (1) the $\{001\}$ pole figure has been determined. Then the ODF was reproduced as a superposition of single orientations on the base of the calculated pole figure (D. I. Nikolaev and T. I. Savyolov, 1987).

If more than ten grains have to be taken into account the occurrence of experimental errors can cause any ambiguity of the ODF reproduction task by $\delta$-function approximation. In this case $f(g)$ must be constructed by Gaussians in the SO(3)-space as described below. For more detail see Savyolova, 1984 and 1985, Nikolayev and Savyolova, 1986, Parthasarathy, 1964 and Perrin, 1928.

\section{APPROXIMATION OF THE ODF BY GAUSSIANS IN THE SO(3)-SPACE}

The main problem of the ODF reproduction from experimental pole figures raises from the lack of information on the odd part of the ODF. Here, the ODF is represented as a superposition of Gaussians. The even part of the desired function should be near to the ODF from the classical harmonics method. The odd component is "added" by the Gaussians. Pole figures which are constructed by such kind of distributions do not contain any information on the odd part. They depend, however, on parameters like weight, peak position and width in the same way as the Gaussian distributions of the rotation group. Therefore, the parameters of the standard distributions can be derived from experimental pole figures directly.

Convenient types of Gaussian distributions of the $\mathrm{SO}(3)$ group are spherical Gaussians (Savyolova, 1984, Nikolayev and Savyolova, 1986):

$$
G\left(g_{0}, \varepsilon, g\right)=\sum_{l=0}^{\infty}(2 l+1) \exp \left\{-l(l+1) \varepsilon^{2}\right\} \sin \{(l+1 / 2) t\} / \sin (t / 2)
$$

with

$$
\cos t=\left(\operatorname{Tr}\left[\left(g_{0}^{*}\right)^{-1} g\right]-1\right) / 2 .
$$

Eq. (9) may be substituted by $\cos (t / 2)=\cos (\beta / 2) \cos \{(\alpha+\gamma) / 2\}$ if $g_{0}=(0,0,0)$ is the origin, where $\{\alpha, \beta, \gamma\}$ are the Euler angles of the rotation $g \in \operatorname{SO}(3)$, and $\varepsilon$ is the dimensionless relative width of the distribution.

The Gaussians for pole figure construction derived from Eq. (8) have the form

$$
P_{\mathbf{h}_{i}}^{G}(\mathbf{y})=\sum_{l=0(2)}^{\infty}(2 l+1) \exp \left\{-l(l+1) \varepsilon^{2}\right\} P_{1}(\cos \tau)
$$

Here, $P_{1}(\cos \tau)$ are the Legendre polynomials with

$$
\cos \tau=\cos \vartheta \cos \chi-\sin \vartheta \sin \chi \cos (\eta-\varphi)
$$


where $\vartheta, \varphi ; \chi, \eta(0 \leq \varphi, \eta<2 \pi ; 0 \leq \vartheta, \chi<\pi)$ are the spherical coordinates of vectors $h_{i}$ and $y$, respectively. For $g_{0} \neq(0,0,0)$ the spherical coordinates $\vartheta^{*}, \varphi^{*}$ of the vector $h_{i}^{*}=g_{0} h_{i}$ have to be used. Terms as described in Eq. (10) are the even components of spherical Gaussians in the $S^{2}$-space (Eq. (8)). The series cut-offs in Eqs. (8) and (10) are chosen in a way that the truncation effects are less then the computer error.

The following algorithm is obtained to determine an ODF $f^{\exp }(g)$ from experimental pole figures $P_{\mathbf{h}_{i}}^{\exp }(\mathbf{y})$.

Step 1: The number of maxima in $f^{\exp }(g)$ and their coordinates $g_{0}^{k}, k=$ $1,2, \ldots, N$ has to be found from the maxima of the function $P_{\mathbf{h}_{i}}^{\exp }(\mathbf{y})$ and their spherical coordinates $\mathbf{y}_{0}^{k}(k=1,2, \ldots, N)$.

Step 2: The ODF $f(g)$ has to be represented as a superposition of spherical Gaussians (Eq. (8)) whose positions are at $g_{0}^{k}$

$$
f(g)=\sum_{k=1}^{N} A_{k} G_{k}\left(g_{0}^{k}, \varepsilon_{k}, g\right)
$$

where $G_{k}\left(g_{0}^{k}, \varepsilon_{k}, g\right)$ is given by Eq. (8) substituting $\varepsilon, t$ by $\varepsilon_{k}, t_{k}$. Taking into account Eq. (10) pole figures may be written as

$$
P_{\mathbf{h}_{i}}^{G}(\mathbf{y})=\sum_{k=1}^{N} A_{k} P_{\mathbf{h}_{i}}^{G}\left(\mathbf{y}, \varepsilon_{k}, g_{0}^{k}\right)
$$

where $P_{\mathbf{h}_{i}}^{G}\left(\mathbf{y}, \varepsilon_{k}, g_{0}^{k}\right)$ is a function of type Eq. (10) and $\cos \left(\tau_{k}\right)$ is the scalar product of $\mathbf{h}_{i}$ and $g_{0}^{k} \cdot \mathbf{y}$.

Step 3: The Gaussian parameters $A_{k}, \varepsilon_{k}$ have to be determined (fit to the experimental data).

If there are isolated maxima in the pole figures the number of distributions and their positions can be found using the method described above. Otherwise, additional conditions have to be taken into account such as a minimum of Gaussians or other considerations. Moreover, the weights of single distributions $A_{k}$ and the widths $\varepsilon_{k}$ are required.

The criterion for the selection of these parameters is the degree of coincidence of experimental and fitted pole figures. In the following two possible approximation methods are discussed.

\section{MEAN ROOT SQUARE APPROXIMATION}

The minimum of the function

$$
L\left(A_{1}, \ldots, A_{N} ; \varepsilon_{1}, \ldots, \varepsilon_{N}\right)=\int_{S^{2}}\left[P_{\mathbf{h}_{i}}^{\exp }(\mathbf{y})-\sum_{k=1}^{N} A_{k} P_{\mathbf{h}_{i}}^{G}\left(\mathbf{y}, \varepsilon_{k}, g_{0}^{k}\right)\right]^{2} d \mathbf{y}
$$

is searched for by means of an iterative procedure. Firstly, starting values of the widths $\varepsilon_{k}^{(0)}(k=1,2, \ldots, N)$ have to be fixed. Differentiating the function $L$ with respect to $A_{k}$ a system of linear equations for $A_{k}^{(i)}$ is obtained. The $i$ th approximation value for $A_{k}$ is found solving this equation system

$$
\sum_{k=1}^{N} A_{k}^{(i)} s_{k l}=r_{l}, \quad l=1,2, \ldots, N
$$


where

$$
\begin{gathered}
s_{k l}=\int_{S^{2}} P_{\mathbf{h}_{i}}^{G}\left(\mathbf{y}, \varepsilon_{k}^{(i-1)}, g_{0}^{k}\right) P_{\mathbf{h}_{i}}^{G}\left(\mathbf{y}, \varepsilon_{l}^{(i-1)}, g_{0}^{l}\right) d \mathbf{y} \\
\mathrm{r}_{l}=\int_{S^{2}} P_{\mathbf{h}_{i}}^{\exp }(\mathbf{y}) P_{\mathbf{h}_{i}}^{G}\left(\mathbf{y}, \varepsilon_{l}^{(i-1)}, g_{0}^{l}\right) d \mathbf{y}
\end{gathered}
$$

Expanding the function $P_{\mathrm{h}_{i}}^{G}\left(\mathbf{y}, \varepsilon_{k}, g_{0}^{k}\right)$ into a Taylor series at the point $\varepsilon_{m}^{(i-1)}$ the following expression is obtained

$$
P_{\mathbf{h}_{i}}^{G}\left(\mathbf{y}, \varepsilon_{m}, g_{0}^{m}\right)=P_{\mathbf{h}_{i}}^{G}\left(\mathbf{y}, \varepsilon_{m}^{(i-1)}, g_{0}^{m}\right)+\left(\Delta \varepsilon_{m}\right)^{2} \frac{\partial}{\partial \varepsilon_{m}^{2}} P_{\mathbf{h}_{i}}^{G}\left(\mathbf{y}, \varepsilon_{m}^{(i-1)}, g_{0}^{m}\right)
$$

Substituting formula (18) into Eq. (14) a system of linear equations for $\Delta \varepsilon_{k}^{2}$ $(k=1, \ldots, N)$

$$
\sum_{k=1}^{N}\left(\Delta \varepsilon_{k}\right)^{2} u_{k m}=v_{m} \quad(m=1, \ldots, N)
$$

is derived after differentiating the function $L$ with respect to $\Delta \varepsilon_{m}^{2}$. The expressions for $u_{k m}$ and $v_{m}$ are

$$
\begin{gathered}
u_{k m}=A_{k}^{(i)} A_{m}^{(i)} \int_{S^{2}} \frac{\partial}{\partial \varepsilon_{m}^{2}} P_{\mathbf{h}_{i}}^{G}\left(\mathbf{y}, \varepsilon_{m}^{(i-1)}, g_{0}^{m}\right) \frac{\partial}{\partial \varepsilon_{k}^{2}} P_{\mathbf{h}_{i}}^{G}\left(\mathbf{y}, \varepsilon_{k}^{(i-1)}, g_{0}^{m}\right) d \mathbf{y} \\
v_{m}=A_{m}^{(i)}\left[\int_{S^{2}} P_{\mathbf{h}_{i}}^{\exp }(y) \frac{\partial}{\partial \varepsilon_{m}^{2}} P_{\mathbf{h}_{i}}^{G}\left(\mathbf{y}, \varepsilon_{m}^{(i-1)}, g_{0}^{m}\right) d \mathbf{y}\right. \\
\left.-\sum_{k=1}^{N} A_{k}^{(i)} \int_{S^{2}} P_{\mathbf{h}_{i}}^{G}\left(\mathbf{y}, \varepsilon_{k}^{(i-1)}, g_{0}^{k}\right) \frac{\partial}{\partial \varepsilon_{m}^{2}} P_{\mathbf{h}_{i}}^{G}\left(\mathbf{y}, \varepsilon_{m}^{(i-1)}, g_{0}^{m}\right) d \mathbf{y}\right]
\end{gathered}
$$

and

$$
\left(\varepsilon_{m}^{(i)}\right)^{2}=\left(\varepsilon_{m}^{(i-1)}\right)^{2}+\left(\Delta \varepsilon_{m}\right)^{2}
$$

From Eq. (20) the linear equation system Eq. (19) is seen to be a symmetric one.

\section{APPROXIMATION IN EVERY POINT}

The Gaussian parameters are determined minimizing the expression

$$
\max _{\mathbf{y} \in S^{2}}\left|P_{\mathbf{h}_{i}}^{\exp }(\mathbf{y})-\sum_{k=1}^{N} A_{k} P_{\mathbf{h}_{i}}^{G}\left(\mathbf{y}, \varepsilon_{k}, g_{0}^{k}\right)\right| \longrightarrow \min
$$

The solution of the problem has been found using the coordinate minimization method. The choice of initial values depends on the structure of the given pole figure. The start approximation for the parameters $g_{0}^{k}$ is found solving the equation

$$
\left[P_{\mathbf{h}_{i}}\left(\mathbf{y}_{1}\right)-P_{\mathbf{h}_{i}}\left(\mathbf{y}_{2}\right)\right]-\left[P_{\mathbf{h}_{i}}^{G}\left(\mathbf{y}_{1}, \varepsilon, g_{0}^{k}\right)-P_{\mathbf{h}_{i}}^{G}\left(\mathbf{y}_{2}, \varepsilon, g_{0}^{k}\right)\right]=0
$$

The coordinates $\mathbf{y}_{1}$ and $\mathbf{y}_{2}$ having a distance of 1 or 2 steps of the measuring mesh are taken in the range of a pole figure maximum. The input for $A_{k}$ is the reciprocal number of pole figure maxima. 
Thus, the ODF can be reproduced from experimental pole figures in an unique way using the described methods. If there is a small number of grains $(N<8-10) f(g)$ can be fitted by $\delta$-functions. For larger $N$ spherical Gaussians have to be used to determine an unambiguous ODF.

\section{NUMERICAL RESULTS-COMPARISON WITH SPHERICAL HARMONICS STUDIES}

The application of the proposed method is demonstrated at the rolling texture of copper. Figure 1 shows the pole figures $\{100\},\{111\}$ and $\{110\}$ being measured at the RFR reactor of the CINR Rossendorf (in the case of $\{100\}$ and $\{110\}$ the second order pole figures were recorded). The pole figures were scanned by an equiangular point mesh (steps of 6 degrees). There are no obviously isolated maxima. Therefore the number and position of peaks is not directly recognizable. For the parameter determination of spherical Gaussians the $\{100\}$ pole figure has been analyzed. This pole figure is characterized by a girdle of high pole density denoted by the "skeleton" line in Figure 2a. The circles represent the traces of possible vector stars (ideal orientations) without consideration of specimen symmetry. Figure $2 \mathrm{~b}$ is derived from Figure $2 \mathrm{a}$ taking into account the orthorhombic symmetry of experimental data. A sufficient pole figure fit was attainable assuming $N=5$ grains as shown in Figure 2c (without specimen symmetry) and in Figure $5 d$ respectively. The orientations $g_{0}^{k}(k=1, \ldots, 5)$ are determined from the vector stars by the methods described above. Besides the girdle there are maxima (denoted by $A$ in Figure $2 \mathrm{~d}$ ) at $\chi=\pi / 2$ and $\varphi=0, \pi$ and somewhat increased pole density at $\varphi=\pi / 2,3 \pi / 2$ refering to the existence of an axial component which is denoted by a dotted line in Figure $2 \mathrm{~d}$. This axial component is not visuable in Figure 1. Its intensity is higher than the pole figure background but less than unity. Therefore, the ODF can be written as

$$
f^{R}(g)=A_{0}+A f^{A}\left(g, \beta_{0}, \gamma_{0}, \varepsilon_{A}\right)+\sum_{k=1}^{5} A_{k} \frac{1}{N_{B}} \sum_{j=1}^{N_{B}=24} G_{k}\left(g, g_{B}, g_{0}^{k}, \varepsilon_{k}\right)
$$

The calculation of the pole figures corresponding to the ODF of Eq. (25) can be performed by

$$
P_{\mathbf{h}_{i}}^{R}(\mathbf{y})=A_{0}+A P_{\mathbf{h}_{i}}^{A}\left(g, \beta_{0}, \gamma_{0}, \varepsilon_{A}\right)+\sum_{k=1}^{5} A_{k} \frac{1}{N_{B}} \sum_{j=1}^{N_{B}} P_{\mathbf{h}_{i}}^{G}\left(\mathbf{y}, \varepsilon_{k}, \mathbf{y}_{0}^{k}\right)
$$

The parameters $Q=\left\{A_{0}, A, A_{1}, \ldots, A_{5} ; \varepsilon_{A}, \varepsilon_{1}, \ldots, \varepsilon_{5}\right\}$ are found using the minimum condition for Eq. (23). In the given case they were assumed to be constant for all orientations $A_{1}=A_{2}=\ldots=A_{5}$ and $\varepsilon_{1}, \varepsilon_{2}=\ldots=\varepsilon_{5}$. The maxima have been determined taking into account 30 points situated in the high pole density range. The calculations were performed in an iterative way. Starting with orientations $g_{0}^{k}$ a parameter set $Q$ is found permitting to correct orientations $g_{0}^{k}$. The iteration procedure has been stopped when the maximum relative deviation between experimental and fitted pole figures was less than $15 \%$.

The pole figures $\{100\},\{111\}$ and $\{110\}$ constructed by means of Eq. (26) from the fitted parameter set are shown in Figure 3 . Because only the $\{100\}$ pole figure 


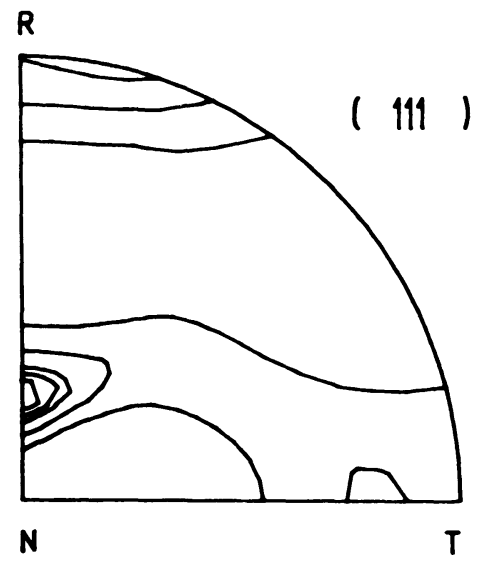

1.002 .003 .003 .504 .00

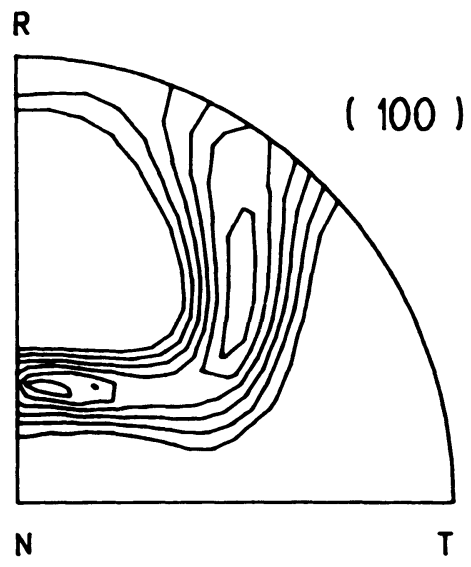

$1.001 .25 \quad 1.50 \quad 1.75 \quad 2.002 .25$

(b)

(a)

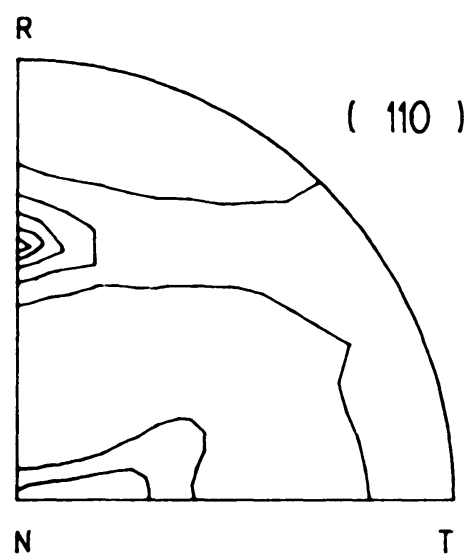

$1.002 .003 .00 \quad 4.00 \quad 4.50$

\section{(c)}

Figure 1 Experimental pole figures. 

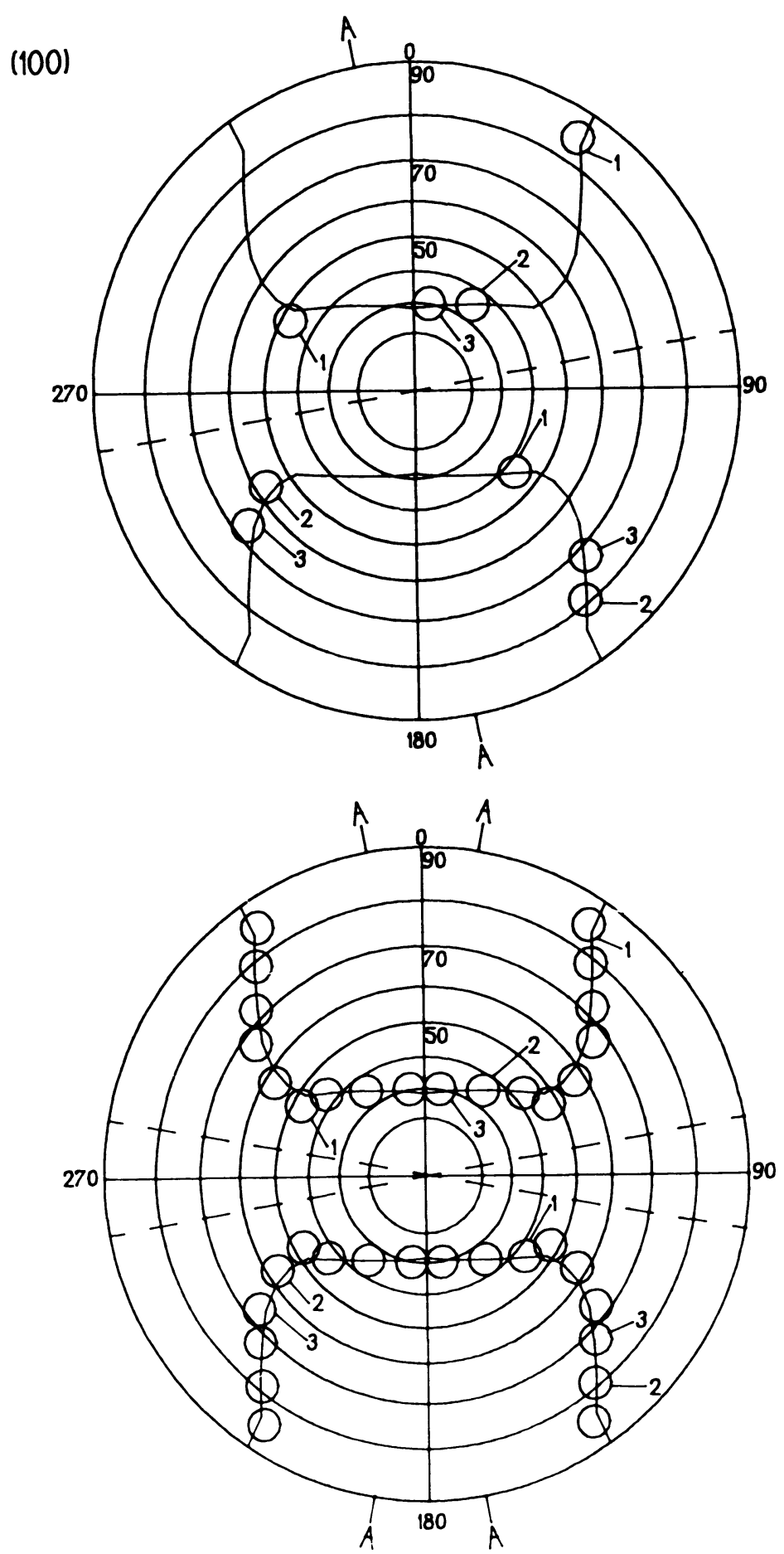

Figure 2 Scheme of the proposed arrangement of vector stars (ideal orientations) in the $\{100\}$ pole figure. The solid line describes the maximum pole density range where the intensity is nearly constant in any perpendicular section. The dotted line represents the assumed position of the axial component. The circles label the exit points of the vector stars. (a)-some possible vector stars (triclinic sample symmetry); (b)-representation of vector stars from (a) taking into account orthorhombic sample symmetry; (c)-position of the fitted vector stars (without specimen symmetry); (d)-representation of vector stars from (c) taking into account orthorhombic specimen symmetry. 

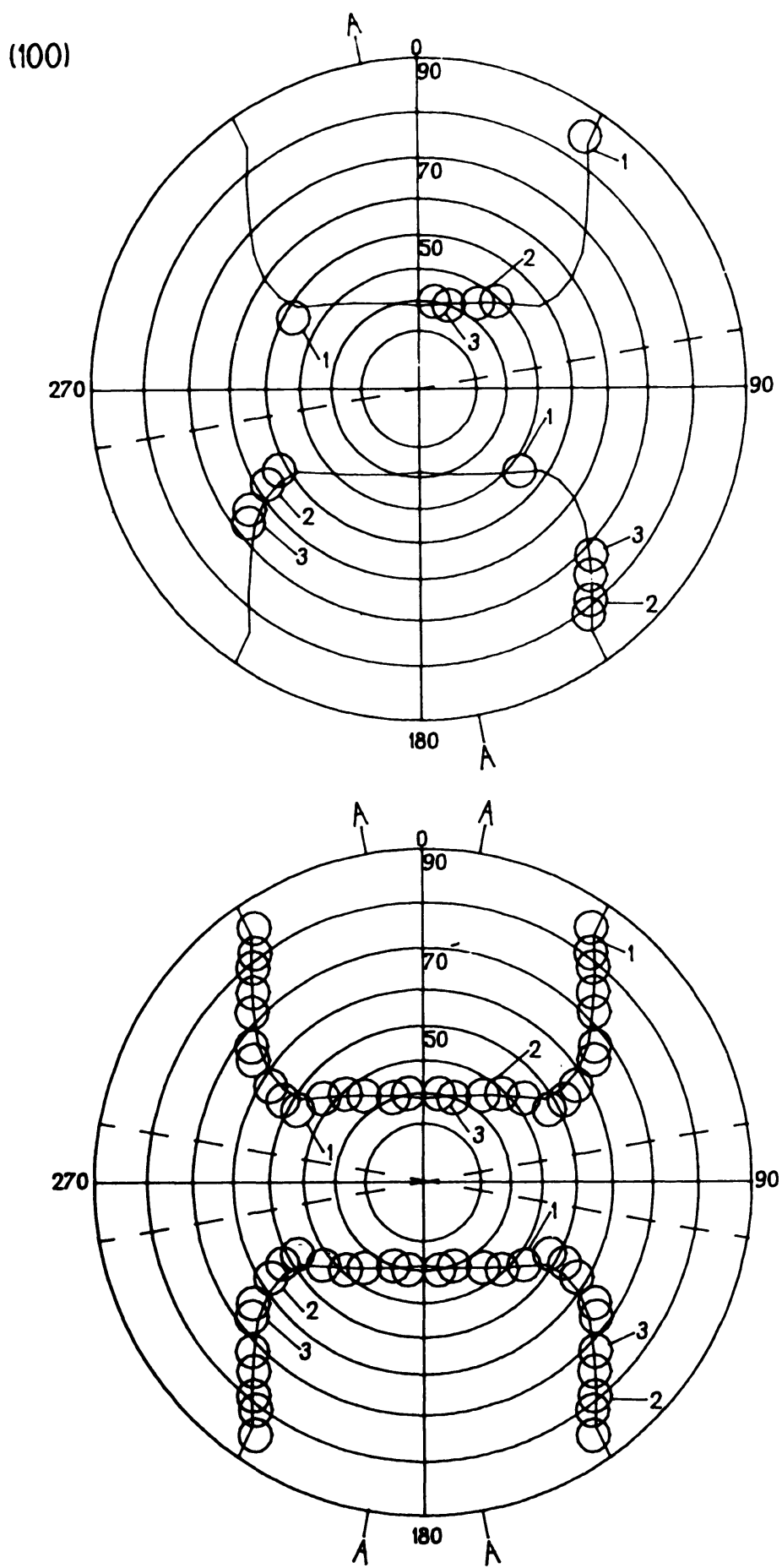

Figure 2 (Continued) Scheme of the proposed arrangement of vector stars (ideal orientations) in the $\{100\}$ pole figure. The solid line describes the maximum pole density range where the intensity is nearly constant in any perpendicular section. The dotted line represents the assumed position of the axial component. The circles label the exit points of the vector stars. (a)-some possible vector stars (triclinic sample symmetry); (b) -representation of vector stars from (a) taking into account orthorhombic sample symmetry; (c)-position of the fitted vector stars (without specimen symmetry); (d)-representation of vector stars from (c) taking into account orthorhombic specimen symmetry. 
APPROXIMATION BY MEANS OF GAUSSIANS

19

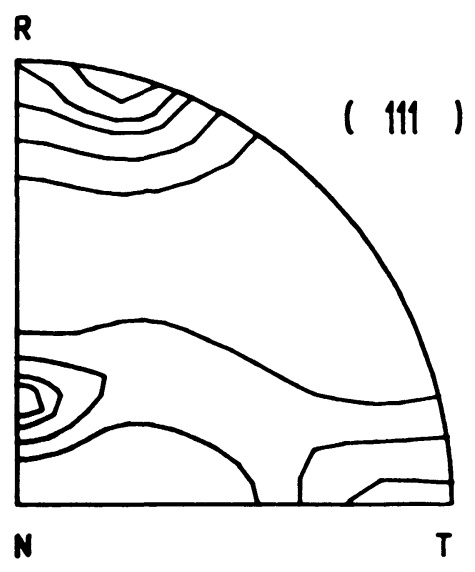

1.002 .003 .003 .504 .00

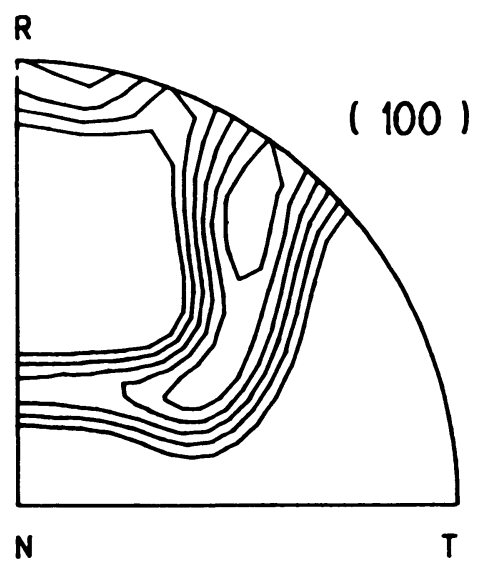

1.001 .251 .501 .752 .002 .25

(a)

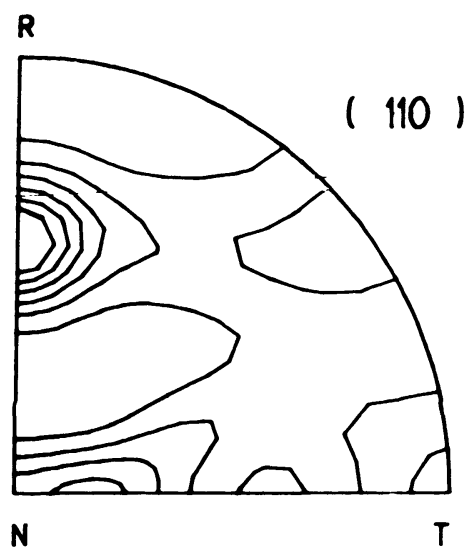

1.002 .003 .004 .004 .50

(c)

Figure 3 Pole figures reproduced by means of the fitted Gaussian parameter set. 
was used for the fit the comparison of experimental and Gaussian $\{111\}$ and $\{110\}$ pole figures may give an idea concerning the efficiency of the proposed method. In Figure 4 the pole figures are shown which are recalculated via spherical harmonics analysis. In Figure 5 constant- $\chi$-sections are given at $\chi=30^{\circ}$ for the experimental $\{100\}$ pole figure, the recalculated one and the Gaussian fitted pole figure as well.

The ODF calculated by means of Eq. (1) is presented in Figure 6. For
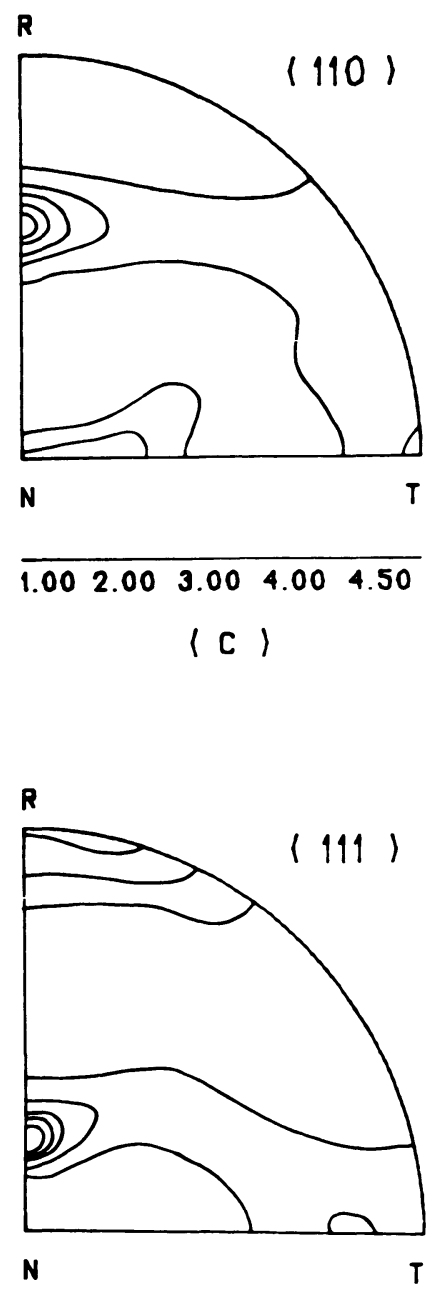

1.002 .003 .003 .504 .00

(b)

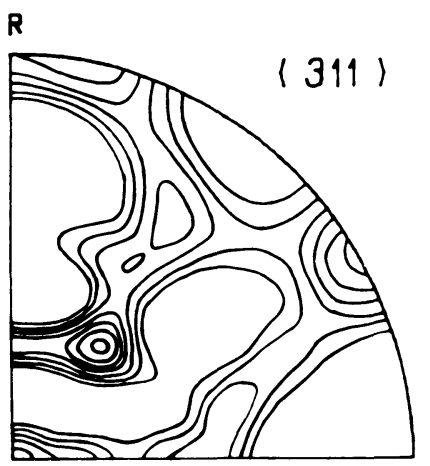

N

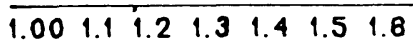

(d)

$\mathbf{R}$

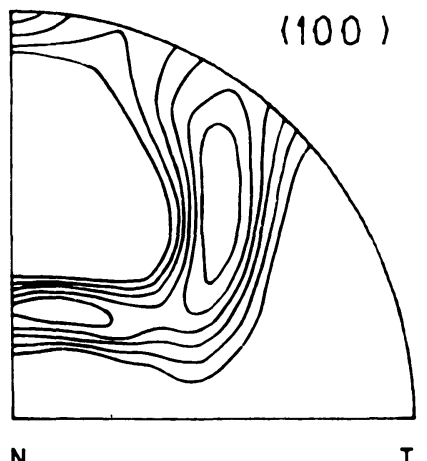

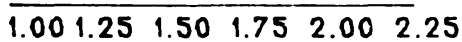

(a)

Figure 4 Pole figures recalculated by means of the spherical harmonics method $\left(1_{\max }=22\right)$. 


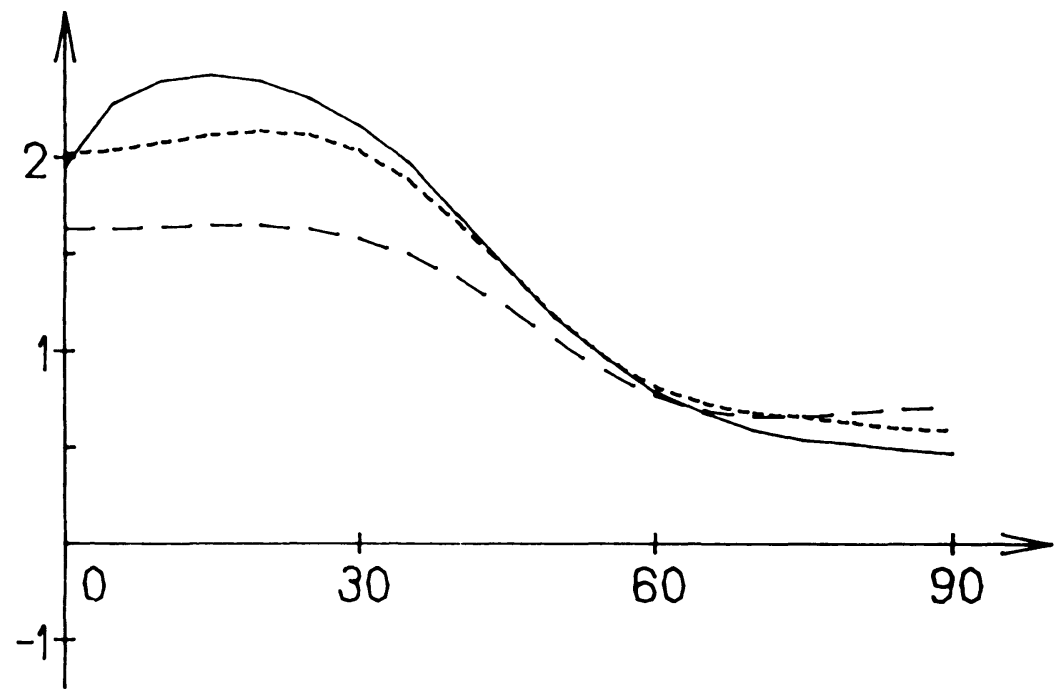

Figure 5 Constant- $\chi$-section of $\{100\}$ pole figures from Figs. 1,3 and 4 as well. The continuous line corresponds to the experimental pole figure, the medium curve to the spherical harmonics recalculated one and the lower dotted line to the Gaussian fitted pole figure respectively.

comparison Figure 7 shows the ODF determined by spherical harmonics analysis. The ODF obtained by the proposed method can be seen to be close to the spherical harmonics one from ODF sections for several constant $\varphi_{2}, \phi$ angles in Figure 8. The even part of the Gaussian ODF deviates from the complete one in the same angular ranges and in the same sense as the spherical harmonics ODF refering to ghost effects. The errors of the proposed method can be estimated by the following characteristics

$$
\begin{gathered}
\left.\sigma^{2}=\int_{S^{2}}\left(P_{\mathbf{h}_{i}}^{\exp }(\mathbf{y})-P_{\mathbf{h}_{i}}^{R}(\mathbf{y})\right)^{2} d \mathbf{y} / \int_{S^{2}} P_{\mathbf{h}_{i}}^{\exp }(\mathbf{y})\right)^{2} d \mathbf{y} \simeq 0.015 \\
R P=100 \% / K \sum_{k=1}^{K} \frac{\left|P_{\mathbf{h}_{i}}^{\exp }(\mathbf{y})-P_{\mathbf{h}_{i}}^{R}(\mathbf{y})\right|}{\left|P_{\mathbf{h}_{i}}^{\exp }(\mathbf{y})\right|} \approx 16 \%
\end{gathered}
$$

Comparing the ODFs from Figures 6 and 7 the maximum of the odd part of the spherical harmonics ODF is 1.01 and the maximum negative value is -0.99 .

The quality of results obtained by the proposed method is essentially determined by the accuracy of the Gaussian parameter fit. It can be estimated comparing the pole figures which were not used for analysis and the corresponding ones reproduced from the fitted parameter set.

\section{CONCLUSION}

In the present paper a method is proposed to reproduce the ODF from pole figures by means of a superposition of Gaussian distributions satisfying the 


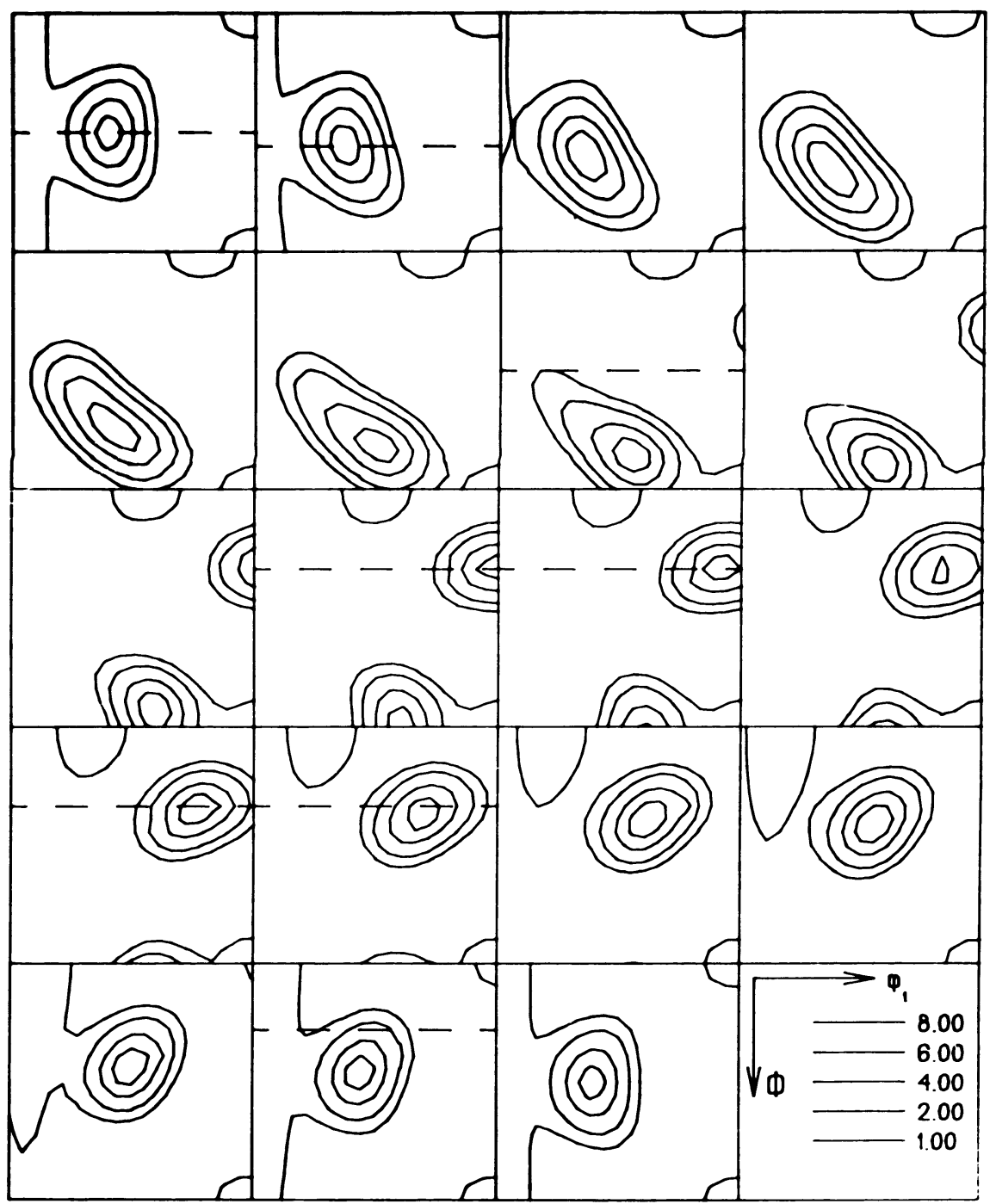

Figure 6 Representation of the Gaussian fitted ODF in $\varphi_{2}=$ const. -sections. The Euler angles in the text and on the Figs. 6 and 7 are related by $\varphi_{1}=\alpha+\pi / 2, \varphi=\beta, \varphi_{2}=\gamma-\pi / 2$.

Central limit theorem. The application of the new formalism is illustrated for an example of the rolling texture of copper.

The representation of the ODF as a superposition of Gaussians fitted in the pole figure space provides the following characteristics and consequences:

-the ODF performs the positivity condition and is free of ghost effects

-1 pole figure is required to determine the ODF by the given method for cubic lattice symmetry, 1 or 2 pole figures are necessary for hexagonal lattices, respectively 


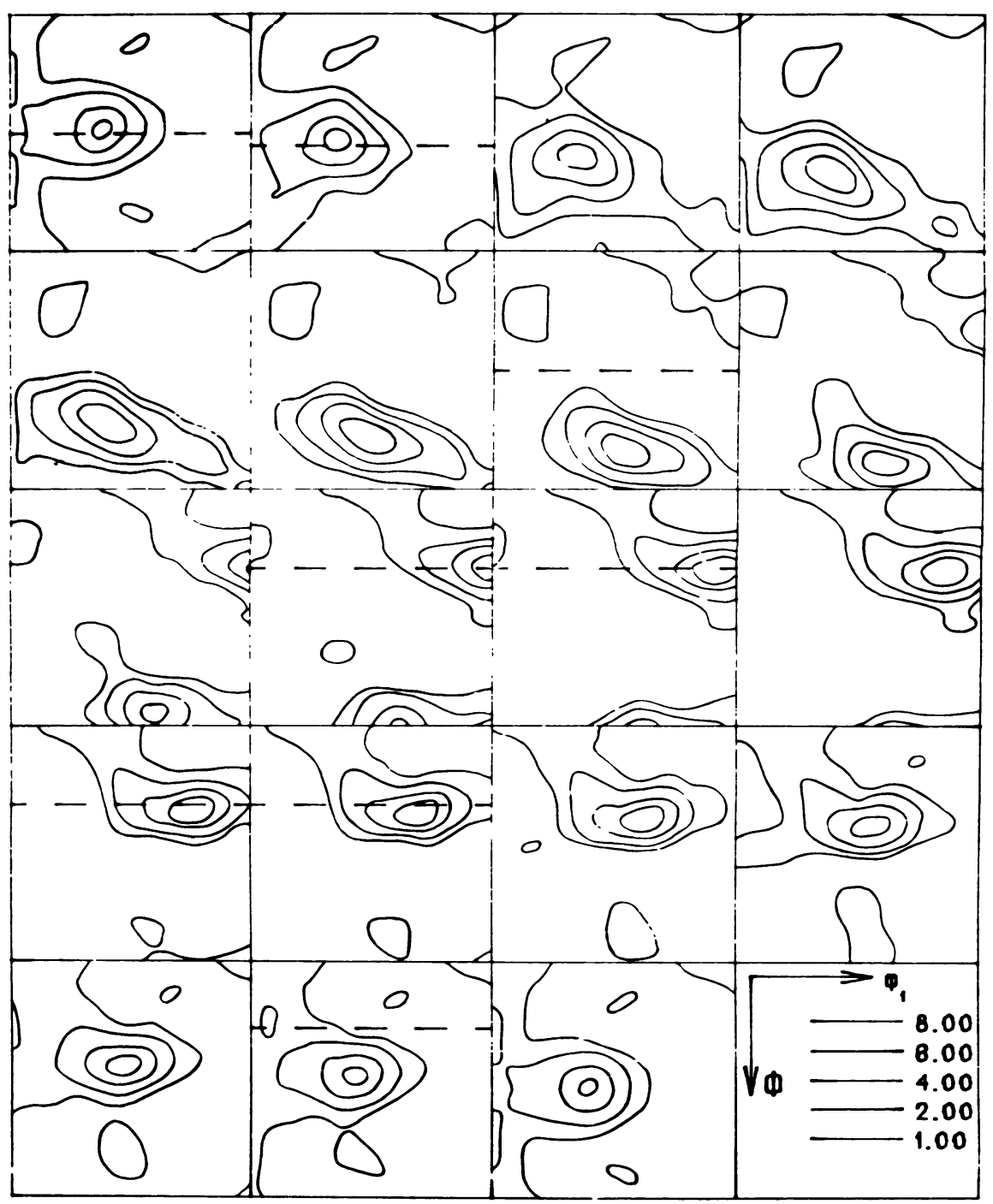

Figure 7 Representation of the ODF in $\varphi_{2}$-const.-sections reproduced by spherical harmonics analysis.

-in the neighbourhood of maxima the ODF is Gaussian shaped if the Central limit theorem is satisfied, i.e. locally the ODF can be considered as a superposition of a large number of random rotations of individual grains.

Up to now, the lack of a simple fully determined approach to find the number and position of Gaussian maxima in the case of overlappings seems to be a drawback of the method. 

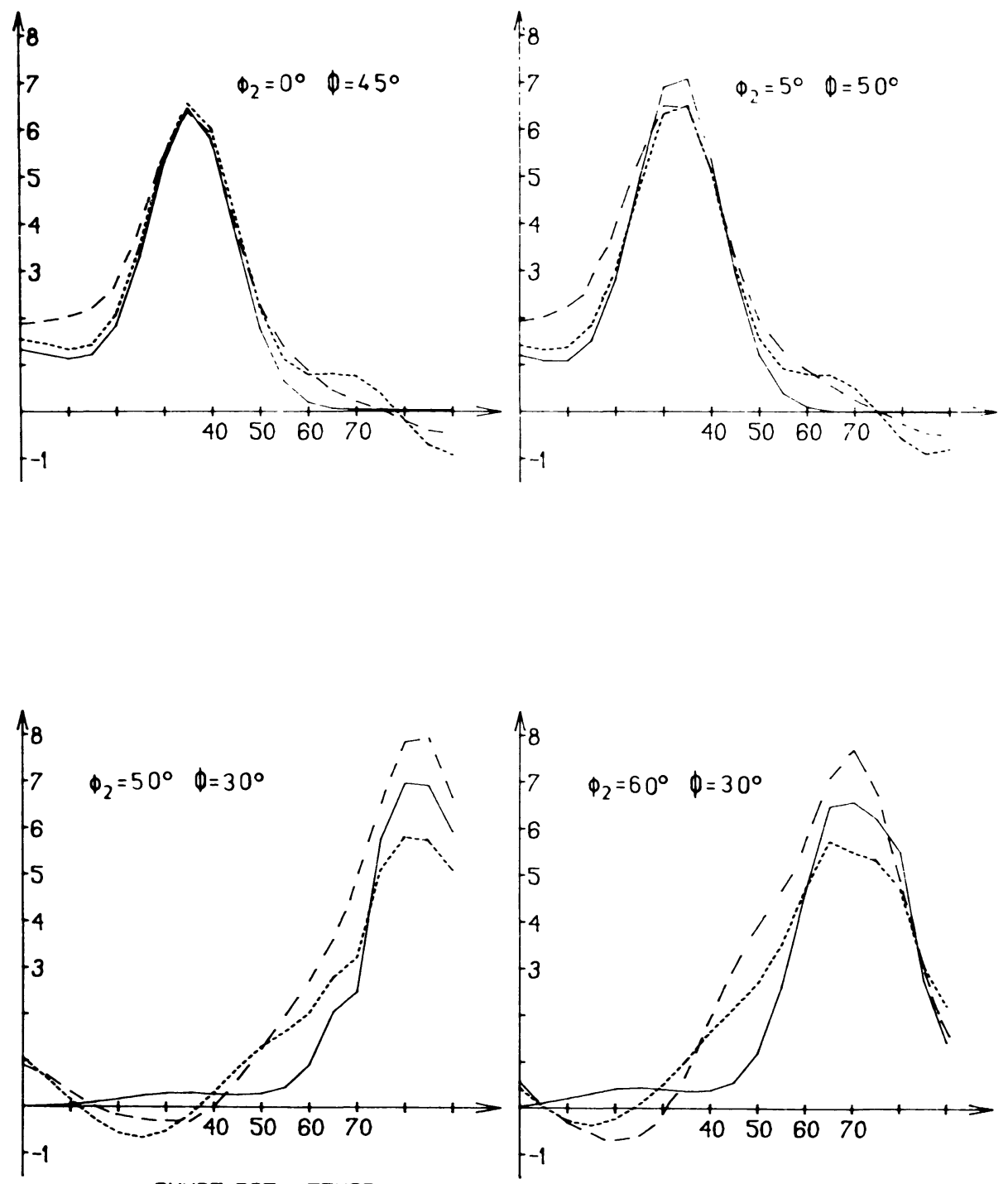

- - - BUNGE-ROE METHOD

- NORMAL DISTRIBUTION

-..-- EVEN PART OF NORMAL DISTRIBUTION

Figure 8 Constant- $\varphi_{2}, \phi$-sections for different angles $\varphi_{2}, \phi$ (in Figure 6a the sections are labelled by dotted lines). 

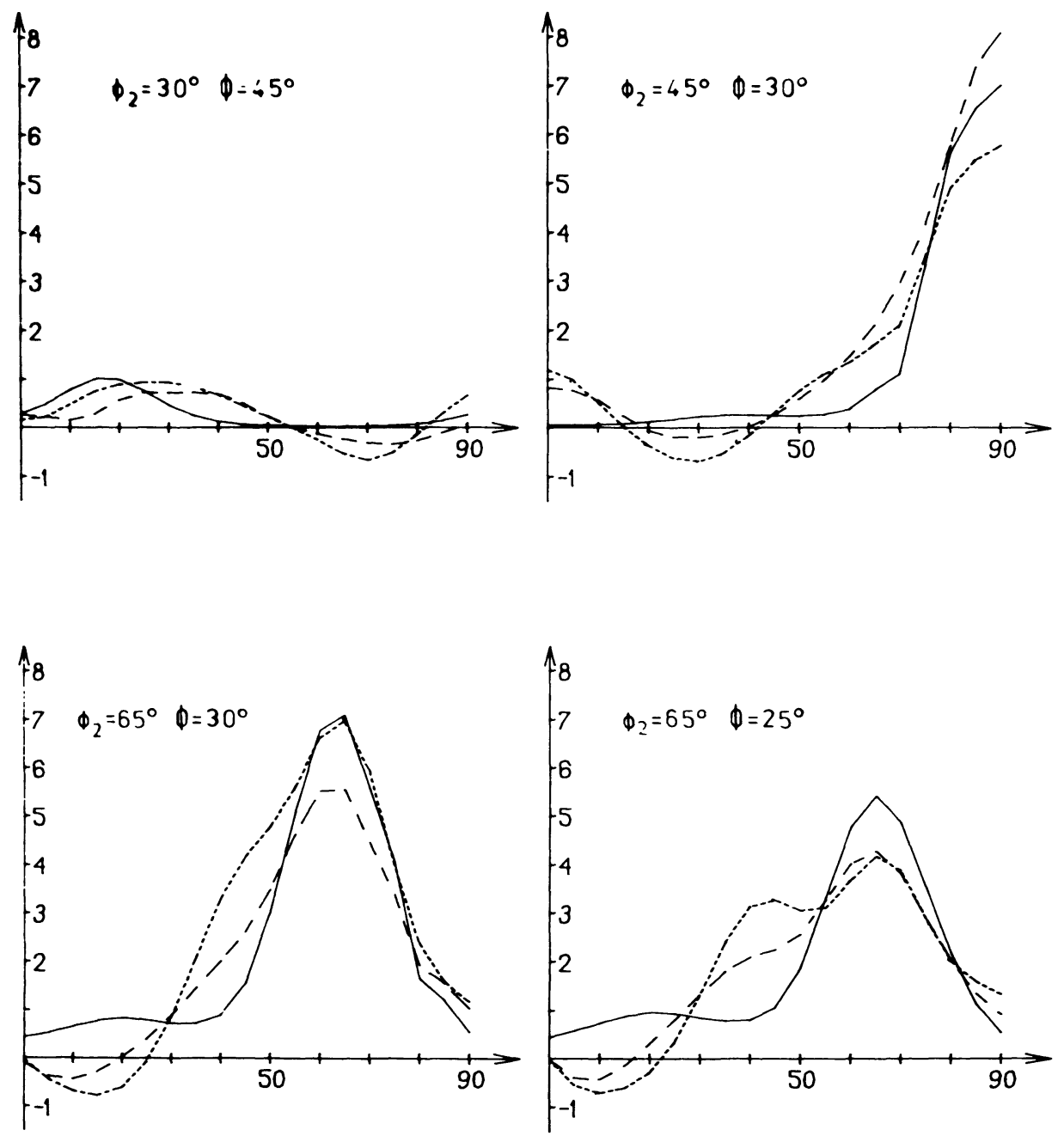

- - -BUNGE-ROE METHOD

- NORMAL DISTRIBUTION

-...-EVEN PART OF NORMAL DISTRIBUTION

Figure 8 (Continued) Constant- $\varphi_{2}, \phi$-sections for different angles $\varphi_{2}, \phi$ (in Figure 6a the sections are labelled by dotted lines). 


\section{ACKNOWLEDGEMENT}

The authors are indebted to Dr. A. Muecklich from CINR Rossendorf for the experimental data and a critical discussion of the manuscript.

\section{APPENDIX}

The scalar product of an ordered vector pair $\mathbf{y}_{1}, \mathbf{y}_{2}$ for the $\{111\}$ pole figure is $\left(y_{1}, y_{2}\right)=1 / 3$. Then, the mapping of vectors as a result of the rotation $g^{*}$ is

$$
\begin{aligned}
& \mathbf{h}_{1}=1 / \sqrt{3}(111) \stackrel{g^{*}}{\longrightarrow} \mathbf{y}_{1}=\left(y_{1}^{1}, y_{1}^{2}, y_{1}^{3}\right) ; \\
& \mathbf{h}_{2}=1 / \sqrt{3}(1 \overline{1} 1) \stackrel{g^{*}}{\longrightarrow} \mathbf{y}_{2}=\left(y_{2}^{1}, y_{2}^{2}, y_{2}^{3}\right) ; \\
& \mathbf{h}_{3}=2 / 3(10 \overline{1}) \stackrel{g^{*}}{\longrightarrow}\left[\mathbf{y}_{1}, \mathbf{y}_{2}\right]=\left(\left[\mathbf{y}_{1}, \mathbf{y}_{2}\right]^{1},\left[\mathbf{y}_{1}, \mathbf{y}_{2}\right]^{2},\left[\mathbf{y}_{1}, \mathbf{y}_{2}\right]^{3}\right)
\end{aligned}
$$

The transformation matrix is

$$
g^{*}=\sqrt{3} / 4\left(\begin{array}{lll}
y_{1}^{1}+y_{2}^{1}+\sqrt{3}\left[y_{1}, y_{2}\right]^{1} & 2\left(y_{1}^{1}-y_{2}^{1}\right) & y_{1}^{1}+y_{2}^{1}-\sqrt{3}\left[\mathbf{y}_{1}, \mathbf{y}_{2}\right]^{1} \\
y_{1}^{2}+y_{2}^{2}+\sqrt{3}\left[y_{1}, y_{2}\right]^{2} & 2\left(y_{1}^{2}-y_{2}^{2}\right) & y_{1}^{2}+y_{2}^{2}-\sqrt{3}\left[\mathbf{y}_{1}, \mathbf{y}_{2}\right]^{2} \\
y_{1}^{3}+y_{2}^{3}+\sqrt{3}\left[y_{1}, y_{2}\right]^{3} & 2\left(y_{1}^{3}-y_{2}^{3}\right) & y_{1}^{3}+y_{2}^{3}-\sqrt{3}\left[\mathbf{y}_{1}, \mathbf{y}_{2}\right]^{3}
\end{array}\right)
$$

Analyzing the $\{110\}$ pole figure the vectors $h_{1}=1 / \sqrt{2}(110)$ and $h_{2}=1 / \sqrt{2}(110)$ are considered. The scalar product of the ordered pair $y_{1}$ and $\mathbf{y}_{2}$ is zero. The relationship between vectors $h_{i}$ and $\mathbf{y}_{i}$ via the rotational operation $g^{*}$ is

$$
\begin{aligned}
& \mathbf{h}_{1}=1 / \sqrt{2}(110) \stackrel{g^{*}}{\longrightarrow} \mathbf{y}_{1}=\left(y_{1}^{1}, y_{1}^{2}, y_{1}^{3}\right) ; \\
& \mathbf{h}_{2}=1 / \sqrt{2}(1 \overline{1} 0) \stackrel{g^{*}}{\longrightarrow} \mathbf{y}_{2}=\left(y_{2}^{1}, y_{2}^{2}, y_{2}^{3}\right) ; \\
& \mathbf{h}_{3}=(00 \overline{1}) \stackrel{g^{*}}{\longrightarrow}\left[\mathbf{y}_{1}, \mathbf{y}_{2}\right]=\left(\left[\mathbf{y}_{1}, \mathbf{y}_{2}\right]^{1},\left[\mathbf{y}_{1}, \mathbf{y}_{2}\right]^{2},\left[\mathbf{y}_{1}, \mathbf{y}_{2}\right]^{3}\right)
\end{aligned}
$$

and the transformation matrix $g^{*}$ is

$$
g^{*}=1 / \sqrt{2}\left(\begin{array}{ccc}
y_{1}^{1}+y_{2}^{1} & y_{1}^{1}-y_{2}^{1} & -\sqrt{2}\left[\mathbf{y}_{1}, \mathbf{y}_{2}\right]^{1} \\
y_{1}^{2}+y_{2}^{2} & y_{1}^{2}-y_{2}^{2} & -\sqrt{2}\left[\mathbf{y}_{1}, \mathbf{y}_{2}\right]^{2} \\
y_{1}^{3}+y_{2}^{3} & y_{1}^{3}+y_{2}^{3} & -\sqrt{2}\left[\mathbf{y}_{1}, \mathbf{y}_{2}\right]^{3}
\end{array}\right)
$$

script.

\section{References}

Bukharova, T. I. and Savyolova, T. I. (1985). Approximation of the Solution of one Inverse Diffraction Problem. Journal of Numerical Mathematics and Mathematical Physics, 25, 617-622, (in Russian). 
Bunge, H. J. (1965). Zur Darstellung allgemeiner Texturen. Zeitschrift fuer Metallkunde, 56, 872-874.

Bunge, H. J. and Esling, C. (1979). Determination of the Odd Part of the Texture Function. Journal Physique Lett., 40, L-627.

Dahms, M. and Bunge, H. J. (1989). The Iterative Series-Expansion Method for Quantitative Texture Analysis. Journal of Applied Crystallography, 22, 439-447.

Luecke, K., Pospiech, J., Virnich, K. H., Jura, J. (1981). On the Problem of the True Orientation Distribution from Pole Figures. Acta Metallurgica, 29, 167.

Matthies, S. (1979). On the Reproducibility of the Orientation Distribution Function of Textured Samples from Pole Figures (Ghost Phenomena). Physica Status Solidi (b), 92, K135-K137.

Matthies, S. (1981). On the Reproducibility of the Orientation Distribution Function of Textured Samples from Pole Figures. Crystal Research and Technology, 16, 513-520.

Nikolaev, D. I. and Savyolova, T. I. (1986). Gaussian Distributions in the SO(3)-Space and their Application for Texture Description. MIFI-Preprint 060-86, Moscow, (in Russian).

Nikolaev, T. I. and Savyolova, T. I. (1987). Approximation of the Solution of one Inverse Diffraction Problem by $\delta$-Functions and Gaussian Distributions. Journal of Numerical Mathematics and Mathematical Physics, 27, 791-793, (in Russian).

Nikolaev, T. I. and Savyolova, T. I. (1987). Numerical Solution of one Inverse Diffraction Problem for a Group of Single Crystals, in: Prikladnye Metody Vytshislityelnoy fiziki. pp. 75-78. Moscow: Energoatomizdat, (in Russian).

Parthasarathy, K. P. (1964). The Central Limit Theorem for the Rotation Group. Theory of Probabilities and its Applications, 9, 273-282.

Perrin, F. (1928). Etude Mathematique du Mouvement Brownien de Rotation. Annales Scientifique de L'ecole Normale Superieure, 45, 1-51.

Savyolova, T. I. (1984). Distribution Function of Grains with Respect to Polycrystal Orientations and its Gaussian Approximation. Zavodskaya Laboratoriya, 50, 48-52, (in Russian).

Savyolova, T. I. (1985). Preface to Novye Metody issledovaniya tekstury polykristallitsheskikh materialov. Sbornik perevodov statyej iz shurnala Texture and Microstructures. Moscow: Metallurgiya, (in Russian). 